\title{
Padrão de distribuição de cupins na região urbana de Goiânia
}

\author{
Diogo A. Costa ${ }^{1}$, Kleber do Espírito Santo Filho² \& Divino Brandão ${ }^{3}$
}

1. Departamento de Ciências Biológicas, Universidade do Estado de Mato Grosso, Caixa Postal 287, 78300-000 Tangará da Serra, MT, Brasil. (diogoacosta@yahoo.com.br)

2. Programa de Doutorado em Ciências Ambientais, Universidade Federal de Goiás, Goiânia, GO, Brasil. (espiritosantok@yahoo.com.br)

3. Departamento de Biologia Geral, Instituto de Ciências Biológicas, Universidade Federal de Goiás, Goiânia, GO, Brasil. (dbrandao@icb.ufg.br)

\begin{abstract}
Distribution patterns of termites on urban region of Goiânia, Goiás, Brazil. A survey of Isoptera pest species in Goiânia, Goiás Brazil about your distribution was related with some environmental factors. A sampling in 600 buildings of different ages located in 20 districts, and the main types of original vegetation was analysed. Four termite species were registered. The analysis showed that the association between infestation frequency and the type of original vegetation has low significance; and between the buildings and districts age has high significance; and neighbouring trees were not an important cause in the distribution pattern of the termites.
\end{abstract}

KEYWORDS. Isoptera, building age, urban fauna, Nasutitermes, Cerrado region.

RESUMO. Foi realizado um levantamento das espécies de cupins em Goiânia, GO, que constituem praga e sua distribuição foi relacionada com alguns fatores ambientais. Realizou-se uma amostragem em 600 edificações de várias idades localizadas em 20 bairros, e analisaramse os principais tipos de vegetação original. Foram registradas quatro espécies de cupins. As análises mostraram que a associação entre a frequência de infestação e o tipo de vegetação original apresentou baixa significância; e entre as idades dos bairros e das edificações apresentou alta significância e constatou-se que árvores próximas não podem ser consideradas uma causa importante do padrão de distribuição dos cupins.

PALAVRAS-CHAVE. Isoptera, idade da edificação, fauna urbana, Nasutitermes, Cerrado.

Existem cerca de 280 espécies de cupins no Brasil (Constantino, 2005), mas apenas uma pequena parcela resiste à urbanização. As características biológicas dos cupins, aliadas à expansão das cidades, têm proporcionado, cada vez mais, a transformação de um maior número de espécies de cupins, cerca de 18, em pragas urbanas (Constantino, 2002). O maior problema tem sido o ataque a móveis, obras de arte, bibliotecas e madeiramento de construção e o controle é geralmente difícil, nem sempre eficaz, sendo mais recomendável adotar medidas preventivas (FonTES, 1995; CostaLEONARDO et al., 2007).

A combinação entre o padrão arquitetônico do ninho, o local onde ele é construído, o tipo de alimento, e o comportamento de forrageamento, diferentes para cada espécie, faz com que as estratégias de controle e/ou de prevenção tenham que ser adequadas para cada espécie de cupim (Fontes \& ARAúJo, 1999; Su \& SCHEFFRAHN, 2000; Fontes \& Milano, 2002; Costa-Leonardo et al., 2007). A identificação correta da espécie é condição essencial para o início do trabalho de controle (MARICONI et al., 1980; Fontes, 1995; Milano, 1998; Costa-Leonardo, 2002; Milano \& FonTes, 2002).

Estudos sobre cupins urbanos no Brasil estão restritos a poucos locais, como na cidade de São Paulo, SP (Fontes, 1995; Lelis, 1995; FerRaZ \& Cancello, 2001; Fontes \& Milano, 2002), Belém, PA (Bandeira, 1998), João Pessoa, PB (Bandeira et al., 1998), Recife, PE (Oliveira et al., 2006), Paulista, PE (Matias et al., 2006), Piracicaba, SP (Eleotério \& Berti Filho, 2000) e Brasília, DF (Constantino \& Dianese, 2001). Em Goiânia, a infestação de cupins nas edificações tem se tornado um problema grave, e as empresas dedicadas ao trabalho de controle frequentemente recorrem à Universidade, na tentativa de identificar as espécies encontradas. No entanto, têm surgido algumas dificuldades nessa identificação, em decorrência do fato de nunca ter sido feito um levantamento preciso das espécies que ocorrem na cidade e, por esse motivo, não se dispor de uma coleção de referência. Além disso, não se dispõe, também, de outras informações, como o padrão de distribuição das várias espécies na área urbana e os fatores que possam explicar o referido padrão. Alguns dados preliminares obtidos a partir de material trazido pela população e coletas esporádicas, sugerem que, entre esses fatores, estariam a localização do bairro e a idade da edificação.

FONTES (1998) afirma que a arborização urbana "constitui reservatório do cupim, dificulta o controle em edificações próximas e favorece re-infestação de áreas tratadas". A arborização das ruas de Goiânia é composta por várias espécies introduzidas e por fragmentos da vegetação original caracterizada por um mosaico onde predominavam dois tipos principais, Cerrado e Floresta de Interflúvio (EITEN, 1994). Embora existam algumas similaridades entre as faunas de cupins dessas duas formações, grande parte das espécies é exclusiva de uma delas (BRAndão \& Souza, 1998; Constantino, 2005; CunHa et al., 2006), e um aspecto interessante dessa diferença está relacionado à proporção de espécies xilófagas (comedoras de madeira), que é muito maior na floresta do que no cerrado (BRANDÃo \& SouZA, 1998).

Os objetivos, do presente estudo, foram realizar um levantamento das espécies de Isoptera que infestam 
madeiramento de construção, móveis, outras peças de madeira, livros e papéis na cidade de Goiânia e relacionar a distribuição das espécies registradas com a idade do bairro, da edificação e tipo da vegetação original da área. Investigar o papel desta vegetação como fonte de onde as colônias se dispersam para as edificações.

\section{MATERIAL E MÉTODOS}

Inicialmente, a cidade de Goiânia foi estratificada em função do tipo da vegetação original (Cerrado e Floresta de Interflúvio) mediante a análise de fotografias aéreas de 1964 aliada às evidências existentes hoje (representadas pelos fragmentos de formações naturais ainda existentes).

Dentro de cada estrato foram selecionados 10 bairros distribuídos ao longo do intervalo total de idades, desde bairros implantados em 1938 (época de fundação da cidade) até 1987 , perfazendo um total de 20 bairros. Em cada um desses bairros foram sorteados 10 logradouros (ruas, praças ou outros) e em cada um deles três edificações (perfazendo um total de 600 edificações). O trabalho foi baseado na edição de 1996 da Planta Urbana de Goiânia, produzida pela Prefeitura Municipal.

As edificações foram visitadas de fevereiro a julho de 1999, e em cada uma a equipe entrevistou o morador e fez uma observação visual, visando obter as seguintes informações: idade da edificação, presença de cupins e tipo do material infestado. Quando houve cupins, uma amostra foi coletada. Em casos especiais (ausência do morador ou recusa de informação) o procedimento amostral foi feito na edificação imediatamente posterior na sequência de numeração da rua.

Um raio de $50 \mathrm{~m}$ em torno de cada uma das 600 edificações foi observado, anotando-se a ocorrência de infestação por cupins nas árvores existentes. Realizouse um teste de $\chi^{2}$ (ZAR, 1999) para verificar a relação entre a frequência de infestação nas edificações e na vegetação próxima.

A associação entre a frequência de infestação por cupins nas edificações e a vegetação original foi submetida a um teste de $\chi^{2}$. Para verificar a relação entre a infestação por cupins e a idade da edificação, primeiramente categorizou-se as idades da edificação em intervalos de 10 anos, para reduzir o erro causado pela imprecisão da informação cedida pelos moradores. Posteriormente, realizou um teste de $\chi^{2}$ entre as frequências de infestação em cada categoria de idade.

A idade do bairro foi verificada no "relatório por data do documento" da Divisão de Denominação de Logradouros da Secretaria de Planejamento do Município de Goiânia. A relação entre a frequência de infestação por cupins e a idade do bairro foi verificada através de uma Análise de Regressão Linear Simples (ZAR, 1999).

Amostras de todas as espécies coletadas estão depositadas na Coleção Termitológica da Universidade Federal de Goiás (UFG).

\section{RESULTADOS}

Quatro espécies de cupins, pertencentes a duas famílias e três gêneros, foram registradas, Coptotermes sp., Heterotermes tenuis (Hagen, 1858), H. longiceps (Snyder, 1924) e Nasutitermes corniger (Motschulsky, 1855). Apenas $H$. longiceps infestava exclusivamente a vegetação; as outras três espécies ocorreram nas edificações, em diferentes materiais, mas todas ocorreram em peças de madeira. Durante a coleta de dados, foram encontrados grânulos fecais que apontam para infestação por Kalotermitidae, porém não foi possível comprovar sua presença devido à necessidade de destruição do material examinado.

O teste de $\chi^{2}$ realizado para verificar a associação entre a frequência de infestação nas edificações e a vegetação original (Tab. I) apontou baixa significância. Para este procedimento foi retirada a espécie $H$. longiceps, por ter sido encontrada infestando apenas a vegetação próxima às edificações. Esse resultado indica que a distribuição dos cupins na cidade de Goiânia não é influenciada pelo tipo de vegetação original da área.

Tanto a idade da edificação como a idade do bairro apresentaram associações significativas com a frequência de infestação (Tab. II, Figs. 1, 2). Um teste de significância mostrou que o valor de $b$ (coeficiente angular) é muito significativo $(\mathrm{F}=6,61 ; \mathrm{p}=0,019)$.

Em todas as 600 edificações amostradas havia árvores em um raio de $50 \mathrm{~m}$, e em 39 casos foi constatada a presença de cupins. Em $20(51 \%)$ destes casos, foi encontrada uma edificação próxima infestada pela mesma espécie. $\mathrm{O}$ teste de $\chi^{2}$ apontou diferenças significativas entre as classes (Tab. III), mas a vegetação parece não interferir na infestação das edificações adjacentes.

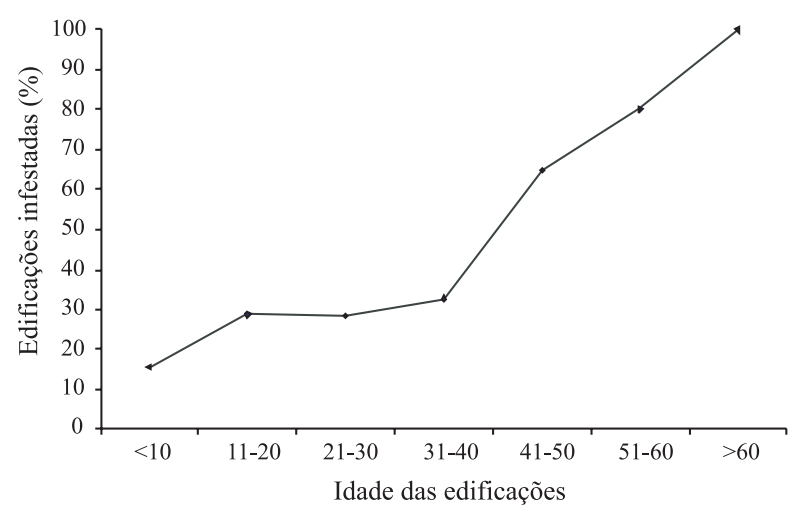

Figura 1. Porcentagem de edificações infestadas de cupins na cidade de Goiânia, GO, de fevereiro a julho de 1999, por intervalo de idade.

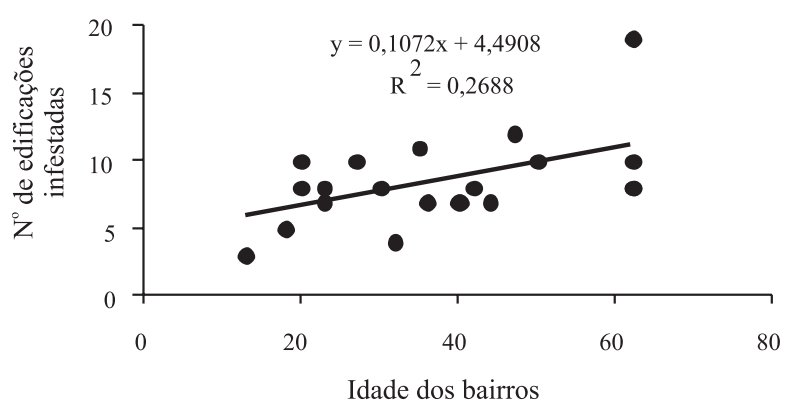

Figura 2. Frequência de infestação de cupins na cidade de Goiânia, $\mathrm{GO}$, de fevereiro a julho de 1999 , em função da idade dos bairros. 
Tabela I. Frequência de infestação em edificações por cupins, verificada de fevereiro a julho de 1999, em Goiânia, GO em relação à vegetação original (entre parênteses as frequências esperadas; $\chi^{2}$ $=3,28(\mathrm{p}=0,350))$.

\begin{tabular}{lccc}
\hline & Infestadas & Não infestadas & Total \\
\hline Floresta & $95(85)$ & $205(215)$ & 300 \\
Cerrado & $75(85)$ & $225(215)$ & 300 \\
Totais & 170 & 430 & 600 \\
\hline
\end{tabular}

Tabela II. Frequência de edificações infestadas por cupins, de fevereiro a julho de 1999, em Goiânia, GO, em relação à idade das edificações em anos (entre parênteses as frequências esperadas); o grupo "indeterminado" não foi considerado para esse cálculo (TE, total de edificações; EI, edificações infestadas; $\chi^{2}=29,23$ $(\mathrm{p}=0,0001))$.

\begin{tabular}{lrr}
\hline Classes de idade & TE & EI \\
\hline$<10$ & 140 & $22(39,67)$ \\
$11-20$ & 174 & $50(49,30)$ \\
$21-30$ & 187 & $53(52,98)$ \\
$31-40$ & 55 & $18(15,58)$ \\
$41-50$ & 17 & $11(4,82)$ \\
$51-60$ & 10 & $8(2,83)$ \\
$>60$ & 2 & $2(0,57)$ \\
indeterminado & 15 & $6(4,25)$ \\
\hline Total & 600 & 170 \\
\hline
\end{tabular}

Tabela III. Frequência de infestações por cupins nas edificações e na vegetação próxima, de fevereiro a julho de 1999, em Goiânia, GO (entre parênteses as frequências esperadas; $\chi^{2}=10,82$ $(\mathrm{p}=0,013))$.

\begin{tabular}{lccc}
\hline & \multicolumn{2}{c}{ Vegetação } & \\
\cline { 2 - 4 } Edificações & Infestada & Não infestada & Total \\
\hline Infestadas & $20(11,05)$ & $150(158,95)$ & 170 \\
Não infestadas & $19(27,95)$ & $411(402,05)$ & 430 \\
\hline Totais & 39 & 561 & 600 \\
\hline
\end{tabular}

\section{DISCUSSÃO}

Entre as espécies de cupins consideradas potencialmente pragas no Brasil (cerca de 18 espécies) (Constantino, 2002), as quatro encontradas no presente estudo até então não haviam sido registradas para Goiânia.

Ao contrário do que se encontra na região Sudeste, onde as principais espécies de cupim-praga são introduzidas (Coptotermes gestroi (Wasmann, 1896) e Cryptotermes brevis (Walker, 1853)) (FonTEs \& Milano, 2002), em Goiânia a espécie mais frequente é $N$. corniger. Situação semelhante foi encontrada no Norte e Nordeste, onde o gênero Nasutitermes (Dudley, 1858) também foi o mais frequente (BANDEIRA, 1998; BANDEIRA et al., 1998; Matias et al., 2006; Oliveira et al., 2006). Além disso, $N$. corniger é considerado uma praga estrutural importante em áreas urbanas em todo Brasil e boa parte da América do Sul, sendo responsável por enormes prejuízos a peças de madeira (Constantino, 2002; Costa-Leonardo, 2002; Fontes \& Milano, 2002; Costa-Leonardo et al., 2007).

A proporção de espécies infestando peças de madeira se assemelha ao encontrado por BANDEIRA (1998) e BANDEIRA et al. (1998) respectivamente em Belém e João Pessoa, onde o material infestado também era preferencialmente madeira, podendo ou não estar associada a qualquer outro tipo de material. Este resultado é plausível, uma vez que todas as espécies de cupins encontradas são xilófagas. Além disso, encontra-se madeira em abundância nas cidades, em particular em Goiânia, onde é um componente importante da construção civil brasileira (COSTA-LEONARDO, 2002; COSTALEONARDO et al., 2007).

Os resultados sugerem uma ligação existente entre a idade da edificação e a infestação por cupins. O envelhecimento dos materiais utilizados na construção, podem ter aumentado a suscetibilidade a infestações, e esta é uma das causas prováveis dessa relação, conforme já salientado por Eleotério \& Berti Filho (2000). A distribuição dos cupins não se dá de maneira aleatória, sendo influenciada também pela idade do bairro. Quanto mais antigo, maior a frequência de infestação, talvez pelo fato de ter havido um maior tempo para a ocupação pelas colônias.

A ocorrência de árvores infestadas próximas de edificações infestadas corrobora a opinião de FonTES (1998) de que pode haver dispersão de colônias entre uma edificação e a vegetação próxima. Entretanto, sua frequência foi apenas de $6,5 \%$ de toda a amostra (contra $28,3 \%$ das edificações). Assim, a reinfestação de uma edificação a partir da vegetação próxima a ela pode não ser uma causa importante do padrão de distribuição da infestação por cupins às edificações em Goiânia.

Agradecimentos. A G. F. Lima Filho, R. A. Carvalho e F. R. Araújo, pelo auxílio no trabalho de campo, a Dra. E. M. Cancello, por facilitar e ajudar durante a visita ao MZUSP para comparação do material, ao Dr. R. Constantino pela identificação das amostras de Nasutitermes, e a Dr. A. M. Costa-Leonardo, pelas sugestões e correção do manuscrito.

\section{REFERÊNCIAS BIBLIOGRÁFICAS}

Bandeira, A. G. 1998. Danos causados por cupins na Amazônia Brasileira. In: Fontes, L. R. \& Berti Filho, E. eds. Cupins. O desafio do conhecimento. Piracicaba, FEALQ. p.87-98.

Bandeira, A. G.; Miranda, C. S. \& Vasconcellos, A. 1998. Danos causados por cupins em João Pessoa, Paraíba - Brasil. In: Fontes, L. R. \& Berti Filho, E. eds. Cupins. O desafio do conhecimento. Piracicaba, FEALQ. p.75-85.

Brandão, D. \& SouZA, R. F. 1998. Effects of deforestation and implantation of pastures on the termite fauna in the Brazilian "Cerrado" region. Tropical Ecology 39:19-22.

Constantino, R. 2002. The pest termites of South America: taxonomy, distribution and status. Journal of Applied Entomology 126:355-365.

2005. Padrões de diversidade e endemismo de térmitas no bioma cerrado. In: Scariot, A. O.; Silva, J. C. S. \& Felfili, J. M. eds. Biodiversidade, Ecologia e Conservação do Cerrado. Brasília, Ministério do Meio Ambiente. p.319-333.

Constantino, R. \& Dianese, E. C. 2001. The urban termite fauna of Brasília, Brazil. Sociobiology 38(3A):323-326.

Costa-Leonardo, A. M. 2002. Cupins-Praga: morfologia, biologia e controle. Rio Claro, Divisa. 128p.

Costa-Leonardo, A. M.; Casarin, F. E. \& Camargo-Dietrich, C. R. R. 2007. Identificação e práticas de manejo de cupins em áreas urbanas. In: Pinto, A. S.; Rossi, M. M. \& SAlmeron, E. orgs. Manejo de pragas urbanas. Piracicaba, CP 2. p.41-54.

Cunha, H. F; Costa, D. A. \& Brandão, D. 2006. Termite (Isoptera) Assemblages in Some Regions of the Goiás State, Brazil. Sociobiology 47(2):1-14. 
Eiten, G. 1994. Vegetação do Cerrado. In: Pinto, M. N. org. Cerrado: caracterização, ocupação e perspectivas. $2^{\text {a }}$ ed., Brasília, SEMATEC/ Ed. UnB. p.17-73.

Eleotério, E. S. R. \& Berti Filho, E. 2000. Levantamento e identificação de cupins (Insecta: Isoptera) em área urbana de Piracicaba - SP. Ciência Florestal 10(1):125-139.

Ferraz, M. V. \& Cancello, E. M. 2001. Swarming behavior of the economically most important termite, Coptotermes havilandi (Isoptera: Rhinotermitidae), in Southeastern Brazil. Sociobiology 38(3):683-693.

Fontes, L. R. 1995. Cupins em áreas urbanas. In: Berti Filho, E. \& Fontes, L. R. eds. Alguns aspectos atuais da biologia e controle de cupins. Piracicaba, FEALQ. p.57-76.

1998. Considerações sobre a complexidade da interação entre o cupim subterrâneo, Coptotermes havilandi, e a arborização no ambiente urbano. In: Fontes, L. R. \& BertI Filho, E. eds. Cupins. O desafio do conhecimento. Piracicaba, FEALQ. p.109-124.

Fontes, L. R. \& AraúJo, R. L. 1999. Os Cupins. In: Mariconi, F. A. $M$. coord. Insetos e outros invasores de residências. Piracicaba, FEALQ. p.35-90.

Fontes, L. R. \& Milano, S. 2002. Termites as an urban problem in South América. Sociobiology 40(1):103-151.

Lelis, A. T. 1995. A nest of Coptotermes havilandi (Isoptera, Rhinotermitidae) off ground level, found in the $20^{\text {th }}$ story of a building in the city of São Paulo, Brazil. Sociobiology 26(3):241-245.

Mariconi, F. A. M.; Zamith, A. P. L.; Araújo, R. L.; Oliveira Filho, A. M. \& Pinchin, R. 1980. Térmitas prejudiciais às madeiras. In: Mariconi, F. A. M.; Zamith, A. P. L.; Araújo, R. L.; Oliveira Filho, A. M. \& Pinchin, R. eds. Inseticidas e seu emprego no combate às pragas. Tomo III: Animais invasores dos domicílios e de outras construções. São Paulo, Livraria Nobel S.A. p.100-123.

Matias, G. R. R. S.; Albuguerque, A. C.; Matias, M. P.; Silva, E. P. V.; Oliveira, C. M. A. S. \& Oliveira, M. A. P. 2006. Os cupins urbanos em Jardim Paulista, Paulista-PE. Diversidade e controle. O Biológico 68(2):58-61.

Milano, S. 1998. Diagnóstico e controle de cupins em áreas urbanas. In: Fontes, L. R. \& Berti Filho, E. eds. Cupins. O desafio do conhecimento. Piracicaba, FEALQ. p.45-74.

Milano, S. \& Fontes, L. R. 2002. Termite pests and their control in urban Brazil. Sociobiology 40(1):163-177.

Oliveira, C. M. A. S.; Matias, G. R. R. S.; Silva, S. B.; Moraes, F. M \& Albuquerque, A. C. 2006. Diversidade de cupins no Ibura: área urbana do Recife-PE. O Biológico 68(2):264-266.

Su, N.-Y. \& Scheffrahn, R. H. 2000. Termites as pest of buildings. In: Abe, T.; Bignell, D. E. \& Higashi, M. eds. Termites: evolution, sociality, symbiosis, ecology. Dordrecht, Kluwer Academic Publishers. p.437-453.

Zar, J. H. 1999. Biostatistical Analysis. Prentice Hall Press, Upper Saddle River. 931p.

Recebido em janeiro de 2008. Aceito em novembro de 2008. ISSN 0073-4721

Artigo disponível em: www.scielo.br/isz 